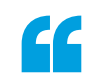

it is possible that this kind

of smart contact lens may be used for the diagnosis and treatment of various diseases in the future
WEARABLE DEVICES

\section{Detect and deliver}

Medical devices to diagnose and treat diseases are of growing interest in healthcare applications. In particular, the on-demand delivery of drugs in a reliable manner is sought after.

Now, Sei Kwang Hahn and colleagues writing in Science Advances report a smart contact lens that monitors glucose concentrations in tears and delivers therapeutic drugs accordingly. The wireless device enables the in vivo treatment of diabetic retinopathy by triggering the delivery of genistein from drug reservoirs within the device.

Contact lenses are a wellestablished medical device making them an attractive starting point for such purposes. The lens forms a

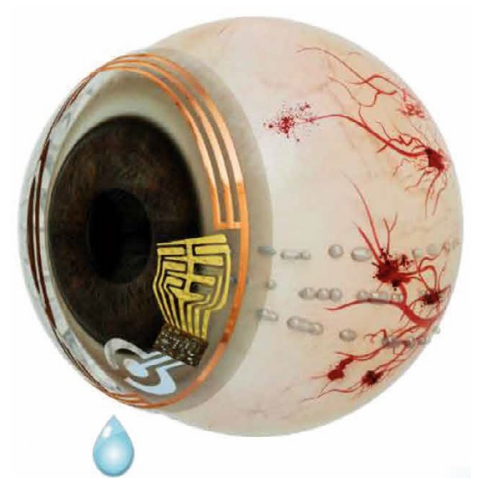

Reprinted with permission of AAAS from Keum, D. H. et al. Sci. Adv. 6, eaba3252 (2020). ${ }^{\circledR}$ The Authors, some rights reserved; exclusive licensee AAAS. Distributed under a Creative Commons Attribution NonCommercial License 4.0 (CC BY-NC). noninvasive interface with the cornea and hence, is a good contact between the device and the body.

"Our smart contact lens contains ultrathin, flexible electrical circuits and a microcontroller chip for realtime electrochemical biosensing, on-demand drug delivery, wireless power management and data communication," says Hahn. The contact lens is composed of silicone hydrogel with a polyethylene terephthalate (PET) film and has a thickness of $200 \mu \mathrm{m}$, a diameter of $14 \mathrm{~mm}$ as well as a radius of curvature of $8 \mathrm{~mm}$.

The electrochemical sensor has three electrodes, with one electrode coated with a solution of glucose oxidase to enable glucose detection. The concentration of glucose in tears identified using this sensor is shown to be consistent with measurements of glucose in blood and hence avoids the need for invasive blood tests.

The drug reservoirs containing genistein are sealed by gold membranes that are dissolved by the application of an electrical current and hence, trigger the release of the loaded drugs. Using fluorescence-labelled genistein, Hahn and colleagues demonstrated that the drug passes through the cornea and sclera, to the retina. Moreover, the delivery of genistein using the smart contact lens is shown to have a similar therapeutic effect in the treatment of diabetic retinopathy compared with the intravitreal injection of the common-drug, Avastin.

The electronic components of the smart contact lens are all remotely controlled by wireless power and communication systems. "More specifically, an external transmitter coil transfers the electrical power to the receiver coil on the contact lens by resonant inductive coupling," explains Hahn. The researchers also show that the thermal stability of the contact lens is sufficient for the material to be used for the purpose of monitoring physiological conditions and delivering drugs.

"Remarkably, changes in our eyes can be directly connected to changes in our liver, lung, heart, brain and kidneys," explains Hahn. "As a result, it is possible that this kind of smart contact lens may be used for the diagnosis and treatment of various diseases in the future, including glaucoma and age-related macular degeneration."

"We are currently working to reduce the thickness of the lens to around $150 \mu \mathrm{m}$, which will enable people to wear it comfortably all the time," says Hahn. Following this step, Hahn envisages moving to clinical trials for the sensing contact lenses.

Alison Stoddart

ORIGINAL ARTICLE Keum, D. H. et al. Wireless smart contact lens for diabetic diagnosis and therapy. Sci. Adv. 6, eaba3252 (2020) 